\title{
SModelS Database Update v1.2.3
}

\author{
Charanjit K. Khosa, ${ }^{1}$ Sabine Kraml, ${ }^{2}$ Andre Lessa, ${ }^{3}$ Philipp Neuhuber, ${ }^{4}$ and Wolfgang Waltenberger ${ }^{4}$ \\ ${ }^{1}$ Department of Physics and Astronomy, University of Sussex, Brighton BN1 9QH, UK \\ ${ }^{2}$ Laboratoire de Physique Subatomique et de Cosmologie, Université Grenoble-Alpes, CNRS/IN2P3, Grenoble INP, \\ 53 Avenue des Martyrs, 38000 Grenoble, France \\ ${ }^{3}$ Centro de Ciências Naturais e Humanas, Universidade Federal do ABC, Santo André, 09210-580 SP, Brazil \\ ${ }^{4}$ Institut für Hochenergiephysik, Österreichische Akademie der Wissenschaften, Nikolsdorfer Gasse 18, 1050 Wien, Austria
}

\begin{abstract}
We present an update of the SModelS database with simplified model results from 13 ATLAS and 10 CMS searches for supersymmetry at Run 2 . This includes 5 ATLAS and 1 CMS analyses for full Run 2 luminosity, i.e., close to $140 \mathrm{fb}^{-1}$ of data. In total, 76 official upper limit and efficiency map results have been added. Moreover, 21 efficiency map results have been produced by us using MadAnalysis5, to improve the coverage of gluino-squark production. The constraining power of the new database, v1.2.3, is compared to that of the previous release, v1.2.2. SModelS v1.2.3 is publicly available and can readily be employed for physics studies.
\end{abstract}

Keywords: physics beyond the standard model, supersymmetry, simplified models, LHC, reinterpretation DOI: $10.31526 /$ LHEP.2020.158

\section{INTRODUCTION}

SModelS $[1,2,3]$ is a public software tool that enables the fast interpretation of simplified model results from ATLAS and CMS searches for supersymmetry (SUSY) in an automatized way. It can be used for evaluating the collider signals of any Beyond the Standard Model (BSM) scenario with a $\mathbb{Z}_{2}$-like symmetry, for which the signal acceptance of the SUSY searches apply [1]. The working principle of SModelS is to decompose all signatures occurring in a given model or scenario into simplified model topologies-also referred to as simplified model spectra (SMS) - by means of a generic procedure where each topology is defined by the vertex structure, the Standard Model (SM), and BSM final states; intermediate $\mathbb{Z}_{2}$-odd BSM particles are characterized only by their masses, production cross sections, and branching ratios.

The signal weights, determined in terms of cross sections times branching ratios, $\sigma \times \mathrm{BR}$, are then matched against a database of LHC results. This is easier and much faster than reproducing analyses with Monte Carlo event simulation, and it allows for reinterpreting searches which are not just cut and count, e.g., analyses which rely on BDT (boosted decision tree) variables. The downside is that the applicability is limited by the simplified model results available in the database. Moreover, whenever the tested signal splits up into many different channels, as often the case in complex models with many new particles, the derived limits tend to be highly conservative. SModelS is thus particularly useful for evaluating constraints and generally characterizing collider signatures in large scans and model surveys.

SModelS makes use of two types of experimental results: upper limit (UL) results and efficiency map (EM) results. Upper limit results provide 95\% confidence level (CL) upper limits on $\sigma \times \mathrm{BR}$ as a function of the respective parameter space of the simplified model-usually BSM masses or slices over mass planes. Their advantage is that the statistical evaluation (i.e., a combination of signal regions when relevant, limit setting pro- cedure, etc.) is done directly by the experimental collaboration. Furthermore, limits obtained from non-cut and count analyses can also be used. However, their statistical interpretation is limited, only allowing for an excluded or not statement, on a purely topology-per-topology basis. Only if the expected UL maps are also available, it becomes possible to select the most sensitive result and/or to compute an approximate likelihood for the signal strength as a truncated Gaussian [4]. Efficiency maps correspond to grids of simulated acceptance times efficiency $(A \times \epsilon)$ values (simply called 'efficiencies' in the following) for the various signal regions of analysis. Their advantage is that they allow for combining contributions from different simplified model topologies to the same signal region, and for computing the likelihood $[2,3]$.

Besides speed, the power of SModelS comes from its large database of results, which is regularly updated. For Run 1, not counting superseded results, the SModelS database contains 93 official UL and 72 EM results from 17 ATLAS and 18 CMS analyses. In [5], we presented the implementation of the Run 2 SUSY search results from CMS with $36 \mathrm{fb}^{-1}$ from the Moriond and the summer (LHCP and EPS) conferences of 2017; this amounted to 84 new UL maps from 19 different analyses. This was further augmented by CMS long-lived particle (HSCP and R-hadron) constraints [6] and the first set of 12 UL maps from six ATLAS SUSY analyses with $36 \mathrm{fb}^{-1}$ in [3]. Moreover, we included 30 "home-grown" EM results relevant for constraining gluino-squark production at $8 \mathrm{TeV}$ [3].

In the new v1.2.3 presented in this paper, this is extended by the simplified model results from 13 new ATLAS and 10 CMS searches for SUSY at Run 2, including the first set of results for full Run 2 luminosity. In total, 76 official UL and EM results have been added. Moreover, 21 EM results (consisting of 351 individual EMs when counting each maps in each signal region separately) for $13 \mathrm{TeV}$ were produced by us for better covering scenarios where gluino-squark associated production is important. In the following, we discuss in detail which results have been added and compare the constraining power of the new database, v1.2.3, to that of the previous release, v1.2.2.

The codebase of SModelS v1.2.3 is almost identical with v1.2.2: apart from two minor bug fixes, the most relevant change is that the database is now by default downloaded from 


\begin{tabular}{|c|c|c|c|c|c|}
\hline Analysis & $\mathcal{L}\left[\mathrm{fb}^{-1}\right]$ & Ref. & ID & SMS results (txnames) & Type \\
\hline 0 lept. + jets & 36.1 & [7] & SUSY-2016-07 & T1, T2, T5WW, T5ZZ, T5WZh, T6WW, T6WZh & UL \\
\hline 0 lept. stop & 36.1 & [8] & SUSY-2016-15 & T2tt, T2bbffff & UL \\
\hline 1 lept. stop & 36.1 & [9] & SUSY-2016-16 & $\mathrm{T}_{2} \mathrm{tt}^{\ddagger}, \mathrm{T} 2 \mathrm{bbffff} f^{\ddagger}, \mathrm{T} 6 \mathrm{bbWW} \mathrm{W}^{\ddagger}$ & UL, EM \\
\hline 2-3 lept. & 36.1 & {$[10]$} & SUSY-2016-24 & $\begin{array}{l}\text { TChiWZ }{ }^{\ddagger} \text {, TSlepSlep } \\
\text { TChiChipmSlepSlep, TChipChimSlepSlep }\end{array}$ & $\begin{array}{l}\text { UL, EM } \\
\text { UL }\end{array}$ \\
\hline photon + jets & 36.1 & {$[11]$} & SUSY-2016-27 & T5gg ${ }^{\ddagger}, \mathrm{T} 5 \mathrm{Zg}, \mathrm{T} 6 \mathrm{gg}^{\ddagger}, \mathrm{TChipChimgg}$ & UL, EM \\
\hline $0-1$ lept. $+b$-jets & 36.1 & [12] & SUSY-2016-28 & $\mathrm{T} 2 \mathrm{bb}$ & UL \\
\hline EW-ino, Higgs & 36.1 & [13] & SUSY-2017-01 & TChiWH & UL \\
\hline Higgsino, $\mathrm{Z} / \mathrm{H}$ & 36.1 & [14] & SUSY-2017-02 & TChiH & UL \\
\hline 2 OS taus & 139.0 & [15] & SUSY-2018-04 & TStauStau & UL \\
\hline 3 lept., EW-inos & 139.0 & [16] & SUSY-2018-06 & TChiWZ & $\mathrm{UL}^{*}$ \\
\hline multi- $b$ & 139.0 & [17] & SUSY-2018-31 & T6bbHH & UL \\
\hline 2 OS lept. & 139.0 & [18] & SUSY-2018-32 & TChiWW, TChipChimSlepSlep, TSlepSlep & UL \\
\hline 1 lept. $+H \rightarrow b \bar{b}$ & 139.0 & [19] & SUSY-2019-08 & TChiWH & UL \\
\hline
\end{tabular}

TABLE 1: Official ATLAS $13 \mathrm{TeV}$ results included in this SModelS database update. All analyses assume large $E_{T}^{\text {miss }}$ in the final state. The 5th column lists the specific SMS results included, using the shorthand "txname" notation (see text for details). For brevity, only the on-shell results are listed, although the off-shell ones are always included (e.g., T2tt in the table effectively means T2tt and T2ttoff; see also [5]). The superscript $\ddagger$ denotes the topologies, for which official EMs from ATLAS are included in addition to the UL maps. The superscript $*$ denotes UL results which contain expected limits in addition to the observed ones.

\begin{tabular}{|c|c|c|c|c|c|}
\hline Analysis & $\mathcal{L}\left[\mathrm{fb}^{-1}\right]$ & Ref. & ID & SMS results (txnames) & Type \\
\hline 0 lept., top tagging & 2.3 & [20] & SUS-16-009 & T1tttt, T2tt, T5tctc & UL* $^{*}$ \\
\hline$\geq 2$ taus & 35.9 & [21] & SUS-17-003 & TChiChipmStauStau, TChipChimStauSnu & UL \\
\hline $\bar{E} W$-ino combination & 35.9 & [22] & SUS-17-004 & TChiWH, TChiWZ & UL \\
\hline 1 lept. compressed stop & 35.9 & [23] & SUS-17-005 & T2bbffff, T6bbWWoff & $\mathrm{UL}^{*}$ \\
\hline jets + boosted $H \rightarrow b \bar{b}$ & 35.9 & [24] & SUS-17-006 & T5HH, T5HZ & $\mathrm{UL}^{*}$ \\
\hline 2 SFOS lept. & 35.9 & [25] & SUS-17-009 & TSelSel, TSmuSmu, TSlepSlep & $\mathrm{UL}^{*}$ \\
\hline 2 lept. stop & 35.9 & [26] & SUS-17-010 & T2tt, T6bbWW, TChipChimSlepSnu & $\mathrm{UL}^{*}$ \\
\hline photon $+(b)$ jets & 35.9 & [27] & SUS-18-002 & T5Hg, T5bbbbZg, T5ttttZg, T6ttZg & $\mathrm{UL}^{*}$ \\
\hline 0 lept. + jets, MHT & 137.0 & [28] & SUS-19-006 & $\mathrm{T} 1, \mathrm{~T} 1 \mathrm{bbbb}, \mathrm{T} 1 \mathrm{tttt}, \mathrm{T} 2, \mathrm{~T} 2 \mathrm{bb}, \mathrm{T} 2 \mathrm{tt}$ & $\mathrm{UL}^{*}$ \\
\hline
\end{tabular}

TABLE 2: Official CMS $13 \mathrm{TeV}$ results included in this SModelS database update; see caption of Table 1 for details.

\begin{tabular}{lcclll} 
Analysis & $\mathcal{L}\left[\mathbf{f b}^{-1}\right]$ & Ref. & ID & SMS results (txnames) & Type \\
\hline 0 lept. + jets & 36.1 & {$[7]$} & ATLAS-SUSY-2016-07 & T1, T2, TGQ, T3GQ, T5GQ, T5WW, T5ZZ, T6WW & EM \\
0 lept. + jets & 35.9 & {$[29]$} & CMS-SUS-16-033 & T1, T1bbbb, T1tttt, T2, T2bb, T2tt, TGQ, T3GQ, T5GQ & EM \\
\hline
\end{tabular}

TABLE 3: Home-grown $13 \mathrm{TeV}$ EM results included in this SModelS database update. Again, for brevity only on-shell results are listed, but the off-shell ones are included as well (this concerns T1tttt, T2tt, T5WW, and T6WW; see [5] for details).

smodels.github.io, contrary to smodels.hephy.at in v1.2.2; see the release notes at https://smodels.readthedocs.io/ en/stable/ReleaseUpdate.html.

Throughout this paper, we assume that the reader is already familiar with SModelS. If this is not the case, the reader may find detailed explanations about the usage and inner workings of SModelS in $[1,2,3]$ and the online manual at https://smodels.readthedocs.io. A detailed discussion of related approaches is given in [30].

\section{NEW RESULTS IN THE DATABASE}

The new (official) ATLAS and CMS results included in the v1.2.3 database are detailed in Tables 1 and 2. They concern all applicable new SUSY search results since SModelS v1.2.2, for which simplified model results are available in digital form on HEPData or the analysis' TWiki page (status end of March 2020). Note that six analyses, five from ATLAS and one from CMS, are for full Run 2 luminosity of about $140 \mathrm{fb}^{-1}$. The home- grown EM results produced by us are listed in Table 3; we will come back to these later.

Inside SModelS, individual SMS results are identified by the analysis ID and the "txname", which describes in a shorthand notation the hypothesized SUSY process (largely following [31]). Due to lack of space, we do not elaborate this naming scheme here, but refer the reader to our "SMS Dictionary" at https://smodels.github.io/docs/ SmsDictionary123, which provides a complete list of txnames together with the corresponding diagrams. Each included map is thoroughly validated to make sure that it reproduces the limits reported in the experimental publication. Detailed validation plots for each result are available online at https: //smodels.github.io/docs/Validation123.

A couple of comments are in order regarding the SMS (UL and EM) results in Tables 1 and 2. First, with the exception of ATLAS-SUSY-2018-06 [16], the UL maps provided by ATLAS contain only the observed limits but not the expected ones. This makes it impossible to deduce the statistically most sensitive 

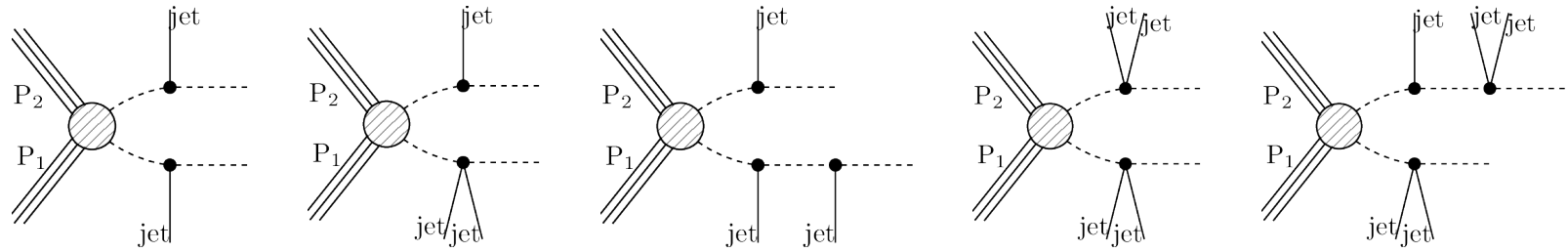

FIGURE 1: Sample of SMS topologies with 2-5 jets relevant to gluino/squark production, for which "home-made" EM results have been produced with the MadAnalysis 5 recast codes $[32,33]$ for the ATLAS and CMS 13 TeV multijet $+E_{T}^{\text {miss }}$ searches $[7,29]$. From left to right: T2, TGQ, T3GQ, T1, and T5GQ.

analysis or to estimate a CL for a given hypothesized signal. On the CMS side, SUS-17-003 and SUS-17-004 do not report expected limits.

Second, while we very highly appreciate the provision of EMs by ATLAS, in many cases, the $A \times \epsilon$ values are for the most sensitive (a.k.a. "best") signal region only. This is not optimal because the best signal region (at a given mass point) can depend on the tested signal and hence vary for different BSM scenarios. We, therefore, want to encourage ATLAS and CMS to provide EMs for all signal regions over the full parameter space of the considered simplified model. In case the number of signal regions is too large, as often the case for CMS searches, this might be done for a set of appropriately aggregated regions.

Third, some EMs provided by ATLAS were not included in the v1.2.3 database because they apply to a sum over SMS topologies instead of a single topology. This is, in particular, the case when mixed decays are assumed, e.g., $\tilde{\chi}_{2}^{0} \rightarrow \tilde{\chi}_{1}^{0}+Z$ or $h^{0}$ with fixed BRs, or $\tilde{\chi}^{ \pm}$decays via sleptons, with the contributions of charged sleptons and sneutrinos summed over. From these combined efficiencies, there is not enough information to infer the contribution from each individual topology. Hence, the result cannot be applied to general models, where the topologies can contribute to different relative weights. Such results are, therefore, not included in SModelS.

A general discussion of simplified model results and recommendations regarding their presentation can be found in Section II.A.5 of the recent report of the LHC Reinterpretation Forum [30].

Concerning the second point above, we note that ATLAS has started to release full likelihoods in the form of a j son serialization [34], which should describe background correlations at the same fidelity as the likelihood model used in the experiment. So far, this is available for two analyses, the sbottom multibottom search [17] (SUSY-2018-31) and the search for direct stau production [15] (SUSY-2018-04), both for full Run 2 luminosity. Both analyses also provide detailed EMs for the simplified models they consider. This is a pioneering step forward in the presentation of BSM searches, which can greatly improve the preservation and reuse of the experimental results. We are currently working on an interface to pyhf [35] to make full use of these data (see contribution no. 15 in [36]). In the meanwhile, the TStauStau and T6bbHH EMs from $[15,17]$ are not included in the new SModelS v1.2.3 database, because using only the best signal regions for limit setting leads to over- or underexclusions compared to the UL results; we think that this is due to background fluctuations and, thus, postpone the usage of the TStauStau and T6bbHH EMs until the full json/pyhf implementation is available in SModelS.

Let us now turn to the home-grown EMs in Table 3. For a good coverage of complex models with several new particles, it is crucial that all major contributions to the total signal considered by a particular analysis can be taken into account. In the SUSY context, this means that a large set of EM results is required in particular for the generic gluino/squark searches; see $[37,38]$. Concretely, it is important to cover topologies arising from gluino-squark associated production in addition to the usual gluino-pair and squark-pair productions [37].

We, therefore, turned to recast the ATLAS and CMS multijet $+E_{T}^{\text {miss }}$ searches $[7,29]$ with MadAnalysis $5[39,40,41]$ in order to produce such a set of EMs for $13 \mathrm{TeV}$ which were then incorporated into the SModelS v1.2.3 database. (Similar homegrown EMs for $8 \mathrm{TeV}$ were already included in v1.2.2 [3].) We considered topologies with 2-5 jets in the final state as shown in Figure 1, to allow for the combination of gluino-pair, squarkpair, and gluino-squark associated production. This was augmented with EMs for additional topologies considered in the experimental searches (T5WW, T5ZZ, and T6WW for the ATLAS analysis; T1bbbb, T1tttt, T2bb, and T2tt for the CMS one) giving the sets listed in Table 3.

For each signal topology, we simulated 10,000 events per parameter point, with the number of parameter points per topology ranging between 251 (CMS-SUS-16-033, T1bbbb) and 2635 (CMS-SUS-16-033 and ATLAS-SUSY-2016-07, TGQ). The total number of parameter points amounts to 22829. We used MadGraph5_aMC@NLO [42] to simulate the hard scattering (with one additional hard jet) processes and Pythia 8 [43] for the decays and parton shower, employing the MLM scheme for matching and merging. The events were then subjected to the MadAnalysis 5 framework, which uses Delphes 3 [44] for emulation of the detector response. The concrete recast codes used were [32] and [33] for the ATLAS and CMS searches, respectively, each with its specific Delphes 3 configuration. Note that [33] employs the aggregate regions of [29], which (as also mentioned in the CMS paper) gives somewhat weaker limits than the full analysis. In a final step, the efficiencies and their relative errors were read from the MadAnalysis 5 output and adapted for SModelS to form a total of 351 individual EMs (22 signal regions $\times 10$ topologies for ATLAS-SUSY-2016-07 and 12 aggregate regions $\times 11$ topologies for CMS-SUS-16-033, minus one which has only zero efficiencies for one topology in one region). For the bulk of the EMs, the statistical uncertainty is typically of the order of $1-10 \%$ for the leading topologies in accordance with the accuracy of the recasting with MadAnalysis 5 . However, it may go up to order $30-50 \%$ in some cases, in particular for very low masses or small mass splittings, where also the Monte Carlo-based recasting is less accurate. Homegrown EMs based on higher statistics will be provided in future releases. 


\section{PHYSICS IMPACT}

We demonstrate the increase in constraining power of SModelS owing to this database update upon the minimal supersymmetric standard model (MSSM) with 19 free parameters defined at the weak scale-the so-called phenomenological MSSM (pMSSM). To this end, we make use of the extensive dataset from the ATLAS pMSSM study [45] available at [46]. Concretely, we use the ATLAS pMSSM scan points with a binolike neutralino as the lightest supersymmetric particle (LSP), which were not excluded by ATLAS at $8 \mathrm{TeV}$. This amounts to about $61.4 \mathrm{~K}$ points with sparticle masses up to $4 \mathrm{TeV}$, of which $28.4 \mathrm{~K}(46 \%)$ are excluded by SModelS v1.2.2. This increases to $34.4 \mathrm{~K}$ excluded points $(56 \%)$ with v1.2.3. ${ }^{1}$ Of the $6 \mathrm{~K}$ newly excluded points, $27 \%$ are excluded only by the home-grown EMs from Table 3. Figure 2 shows the number of excluded points as functions of the gluino, lightest squark, and LSP masses. The histograms on the left illustrate the increase in constraining power from SModelS v1.2.2 to v1.2.3, and the histograms on the right show the relevance of the home-grown EMs in v1.2.3.

SModelS reports its results in the form of $r$-values, defined as the ratio of the theory prediction over the observed upper limit, for each experimental constraint that is matched in the database. All points for which at least one $r$-value equals or exceeds unity $\left(r_{\max } \geq 1\right)$ are considered as excluded. It is instructive to see which analyses/results, thus, turn out as the most constraining ones. For the $6 \mathrm{~K}$ points newly excluded with v1.2.3, the highest $r$-value comes in $51 \%$ of the cases from CMS-SUS-19-006 (mostly the T2 ULs), followed by ATLAS-SUSY-2016-07 EMs (36\%) and ATLAS-SUSY-2018-32 (6\%, mostly TSlepSlep ULs).

The importance of the combination of contributions from the topologies shown in Figure 1 is best shown upon an explicit example. To this end, we choose 424686577 . slha from the ATLAS pMSSM dataset [46]. This point features heavy squarks $\left(m_{\tilde{q}} \sim 2.5-3.5 \mathrm{TeV}\right)$, a light gluino $\left(m_{\tilde{g}} \simeq 976 \mathrm{GeV}\right)$, and an LSP which is close in mass to the gluino $\left(m_{\tilde{\chi}_{1}^{0}} \simeq 908 \mathrm{GeV}\right)$. The gluino decays to the LSP via two competing modes, 3-body decays into the LSP plus two quarks (BR $\simeq 40 \%$ for $q \bar{q} \tilde{\chi}_{1}^{0}, 16 \%$ for $b \bar{b} \tilde{\chi}_{1}^{0}$ ) or loop decays into the LSP plus a gluon (BR $\simeq 44 \%$ ). As a result, the signal of gluino-pair production consists of $16 \% \mathrm{~T} 1$, $19 \%$ T2, and 35\% TGQ. The heavy squarks, on the other hand, decay into the gluino plus a quark with BRs of 90-99\%. Thus, gluino-squark associated production generates the T3GQ and T5GQ topologies from Figure 1 (the contribution from pair production of squarks is subdominant). Table 4 lists the individual contributions of each topology to the total signal for the best signal region of the CMS-SUS-16-033 analysis. As we can see, each individual topology contributes with an $r$-value less than 0.3 , and only the combination of all contributions allows SModelS to exclude this particular point. This is only possible due to the efficiency maps listed in Table 3.

\section{USAGE}

The new database presented here is shipped with the SModelS v1.2.3 package, but it can also be used with the earlier v1.2.x code versions. The easiest way is by specifying the path

${ }^{1}$ For comparison, with the update from v1.1.2 [5] to v1.2.2, the number of excluded points increased only by about $2 \%$.

\begin{tabular}{|c|c|c|}
\hline Topology & SUSY process(es) & $\begin{array}{c}\text { Contribution } \\
\text { to } r \text {-value }\end{array}$ \\
\hline T1 & $p p \rightarrow \tilde{g} \tilde{g} \rightarrow q q \tilde{\chi}_{1}^{0}+q q \tilde{\chi}_{1}^{0}$ & 0.16 \\
\hline T2 & $p p \rightarrow \tilde{g} \tilde{g} \rightarrow g \tilde{\chi}_{1}^{0}+g \tilde{\chi}_{1}^{0}$ & 0.18 \\
\hline TGQ & $p p \rightarrow \tilde{g} \tilde{g} \rightarrow g \tilde{\chi}_{1}^{0}+q q \tilde{\chi}_{1}^{0}$ & 0.17 \\
\hline T3GQ & $p p \rightarrow \tilde{g} \tilde{q} \rightarrow g \tilde{\chi}_{1}^{0}+q \tilde{g}\left(\rightarrow g \tilde{\chi}_{1}^{0}\right)$ & 0.31 \\
\hline T5GQ & $p p \rightarrow \tilde{g} \tilde{q} \rightarrow q q \tilde{\chi}_{1}^{0}+q \tilde{g}\left(\rightarrow q q \tilde{\chi}_{1}^{0}\right)$ & 0.27 \\
\hline \hline Total & $\tilde{g} \tilde{g}, \tilde{g} \tilde{q}$ production & 1.09 \\
\hline
\end{tabular}

TABLE 4: Contributions from each individual SMS topology to the total $r$-value for the pMSSM point 424686577 discussed in the text. They correspond to the ratio between the signal yield and the observed upper limit for the signal region $N_{j e t}=$ $5, N_{b}=0, H_{T}>1500 \mathrm{GeV}$, and $H_{T}^{\text {miss }}>750 \mathrm{GeV}$ from CMSSUS-16-033.

to the database URL. When using runSModelS.py, this means setting

path $=$ http://smodels.github.io/database/official123

in the parameters file. In SModelS v1.2.3, also the shorthand notation path = official can be used. When writing one's own python main program, one has to set

database $=$ Database $(" \ldots ")$

where the dots stand for the same database URL as above. The official, precompiled pickle file official123.pcl (680 MB) is then downloaded upon first execution. Note that this download is often faster than parsing the text database oneself.

Users who want to update the text database in an existing SModelS v1.2.x installation, can download the .zip or .tar.gz file from https://github.com/SModelS/smodels-database-release/ releases. It suffices to put this tarball into the main smodels folder and explode it there. That is, the following steps need to be performed:

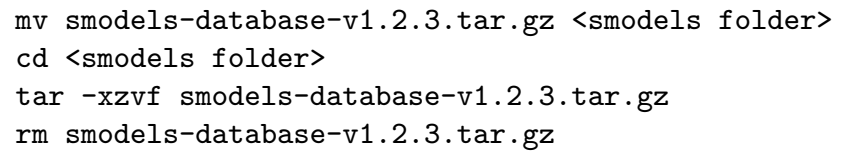

The new database will be unpacked into the smodels-database directory, replacing the previous version, and the pickle file will then be automatically rebuilt on the next run of SModelS. For a clean installation, it is recommended to first remove the previous database version. If the tarball is unpacked to another location, one has to correctly set the SModelS database path when running SModelS. If using runSModelS.py, this is done in the parameters.ini file.

\section{CONCLUSIONS}

We presented the update of the SModelS database with the simplified model results from 13 ATLAS and 10 CMS SUSY analyses from Run 2 with $36-139 \mathrm{fb}^{-1}$ of data. This comprises 76 new official UL and EM results from ATLAS and CMS, supplemented by 21 EM results produced by us using MadAnalysis5. These results significantly improve previously available constraints. 

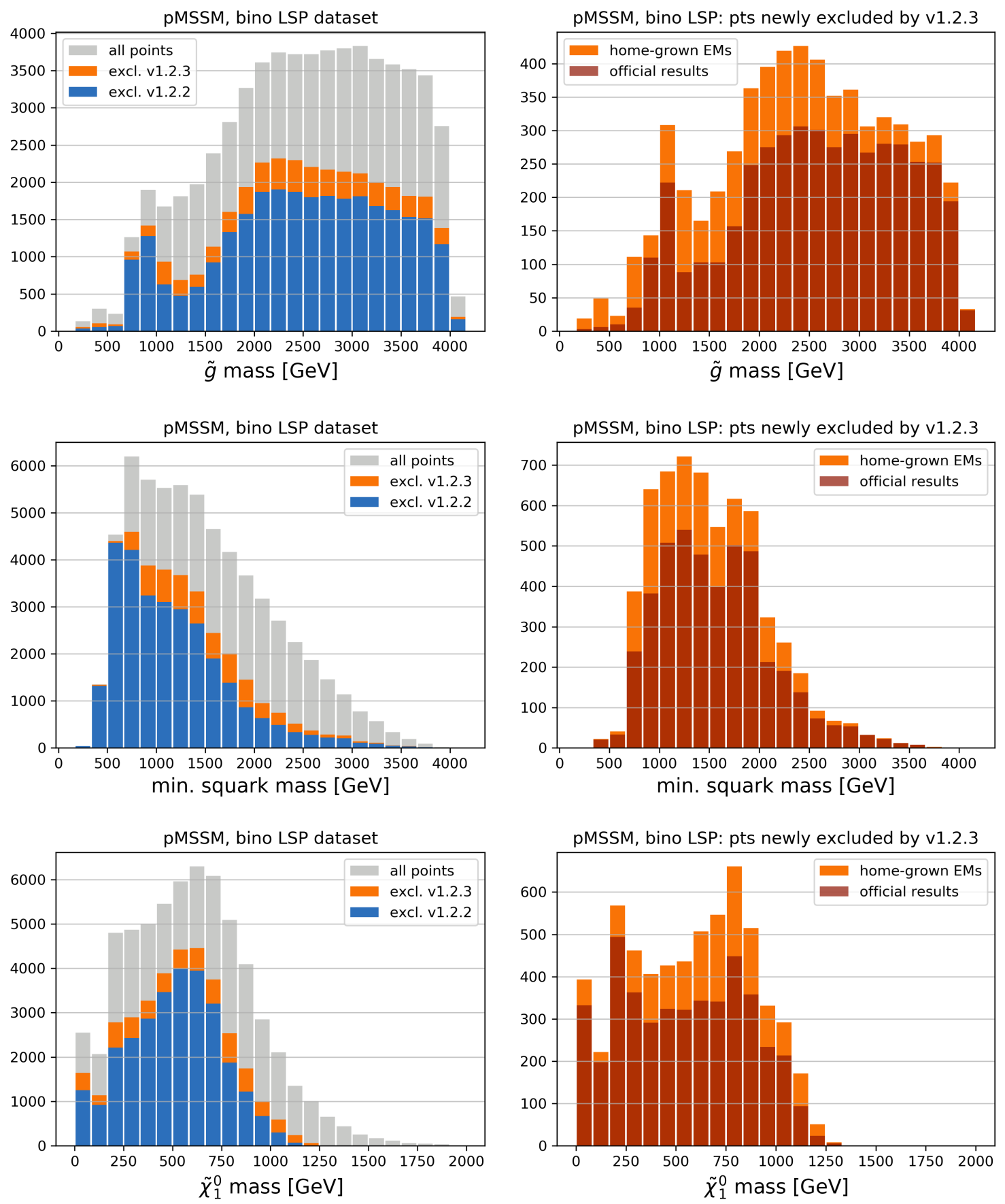

FIGURE 2: Results for the ATLAS pMSSM points with a bino-like LSP, which were classified as not excluded in [45], as function of the mass of the gluino (top row), lightest squark (middle row), and $\tilde{\chi}_{1}^{0}$ LSP (bottom row). The histograms on the left show the number of points excluded by SModelS v1.2.2 (blue) and the number of additional points excluded with the v1.2.3 update (orange); the distribution of all points in the dataset is shown in grey. The histograms on the right detail the number of newly excluded points when using only the official ATLAS and CMS results in v1.2.3 (dark red) as compared to using the full v1.2.3 database including the home-grown efficiency maps (orange). 
In total, the SModelS v1.2.3 database now contains $170 \mathrm{UL}$ and 42 EM results ${ }^{2}$ from 23 ATLAS and 29 CMS analyses at $13 \mathrm{TeV}$, plus a large number of results for $8 \mathrm{TeV}$. The SModelS package is publicly available and can readily be used to constrain arbitrary BSM models which have a $\mathbb{Z}_{2}$ symmetry, provided that the SMS assumptions [1,2] apply. We will continue to update it as new ATLAS and CMS search results become available.

Simplified models have become one of the standard methods for ATLAS and CMS to communicate the results of their searches for new particles. In order to maximize the usefulness of this approach, we encourage the experimental collaborations to provide expected ULs in addition to the observed ones, to provide EMs for all signal regions over the full parameter space of the considered simplified model and generally to provide results for single SMS topologies rather than a combination of two or more topologies. Information enabling the combination of SRs, i.e., full or simplified likelihoods, is also highly valuable. Detailed arguments and recommendations for the presentation of simplified model results are elaborated in Section II.A.5 of the LHC Reinterpretation Forum report [30].

\section{ACKNOWLEDGMENTS}

We thank the ATLAS and CMS SUSY groups for providing a vast number of simplified model results in a digital form. This work was supported in part by the IN2P3 project "Théorie - BSMGA". C.K.K. acknowledges the support from the Royal Society-SERB Newton International Fellowship (NF171488). The work of A. L. was supported by the São Paulo Research Foundation (FAPESP), project 2015/20570-1.

\section{References}

[1] S. Kraml, S. Kulkarni, U. Laa, A. Lessa, W. Magerl, D. Proschofsky, and W. Waltenberger, "SModelS: a tool for interpreting simplified-model results from the LHC and its application to supersymmetry," Eur.Phys.J. C74 (2014) 2868, 1312.4175.

[2] F. Ambrogi, S. Kraml, S. Kulkarni, U. Laa, A. Lessa, V. Magerl, J. Sonneveld, M. Traub, and W. Waltenberger, "SModelS v1.1 user manual: Improving simplified model constraints with efficiency maps," Comput. Phys. Commun. 227 (2018) 72-98, 1701.06586.

[3] F. Ambrogi et al., "SModelS v1.2: long-lived particles, combination of signal regions, and other novelties," 1811.10624.

[4] A. Azatov, R. Contino, and J. Galloway, "Model-Independent Bounds on a Light Higgs," JHEP 04 (2012) 127, 1202. 3415. [Erratum: JHEP 04, 140 (2013)].

[5] J. Dutta, S. Kraml, A. Lessa, and W. Waltenberger, "SModelS extension with the CMS supersymmetry search results from Run 2," LHEP 1 (2018), no. 1, 5-12, 1803.02204.

[6] J. Heisig, S. Kraml, and A. Lessa, “Constraining new physics with searches for long-lived particles: Implementation into SModelS," Phys. Lett. B788 (2019) 87-95, 1808.05229.

\footnotetext{
${ }^{2}$ The 42 EM results consist in fact of 458 individual maps in the different signal regions of 6 ATLAS and 3 CMS analyses.
}

[7] ATLAS Collaboration, M. Aaboud et al., "Search for squarks and gluinos in final states with jets and missing transverse momentum using $36 \mathrm{fb}^{-1}$ of $\sqrt{\mathrm{s}}=13 \mathrm{TeV} \mathrm{pp}$ collision data with the ATLAS detector," Phys. Rev. D97 (2018), no. 11, 112001, 1712.02332.

[8] ATLAS Collaboration, M. Aaboud et al., "Search for a scalar partner of the top quark in the jets plus missing transverse momentum final state at $\sqrt{s}=13 \mathrm{TeV}$ with the ATLAS detector," JHEP 12 (2017) 085, 1709. 04183.

[9] ATLAS Collaboration, M. Aaboud et al., "Search for top-squark pair production in final states with one lepton, jets, and missing transverse momentum using 36 $\mathrm{fb}^{-1}$ of $\sqrt{s}=13 \mathrm{TeV}$ pp collision data with the ATLAS detector," JHEP 06 (2018) 108, 1711.11520.

[10] ATLAS Collaboration, M. Aaboud et al., "Search for electroweak production of supersymmetric particles in final states with two or three leptons at $\sqrt{s}=13 \mathrm{TeV}$ with the ATLAS detector," Eur. Phys. J. C78 (2018), no. 12, 995, 1803.02762.

[11] ATLAS Collaboration, M. Aaboud et al., "Search for photonic signatures of gauge-mediated supersymmetry in $13 \mathrm{TeV} p p$ collisions with the ATLAS detector," Phys. Rev. D97 (2018), no. 9, 092006, 1802.03158.

[12] ATLAS Collaboration, M. Aaboud et al., "Search for supersymmetry in events with $b$-tagged jets and missing transverse momentum in $p p$ collisions at $\sqrt{s}=13 \mathrm{TeV}$ with the ATLAS detector," JHEP 11 (2017) 195, 1708.09266.

[13] ATLAS Collaboration, M. Aaboud et al., "Search for chargino and neutralino production in final states with a Higgs boson and missing transverse momentum at $\sqrt{s}=13 \mathrm{TeV}$ with the ATLAS detector," Phys. Rev. D100 (2019), no. 1, 012006, 1812.09432.

[14] ATLAS Collaboration, M. Aaboud et al., "Search for pair production of higgsinos in final states with at least three $b$-tagged jets in $\sqrt{s}=13 \mathrm{TeV} p p$ collisions using the ATLAS detector," Phys. Rev. D98 (2018), no. 9, 092002, 1806.04030.

[15] ATLAS Collaboration, G. Aad et al., "Search for direct stau production in events with two hadronic $\tau$-leptons in $\sqrt{s}=13 \mathrm{TeV} p p$ collisions with the ATLAS detector," Phys. Rev. D101 (2020), no. 3, 032009, 1911.06660.

[16] ATLAS Collaboration, G. Aad et al., "Search for chargino-neutralino production with mass splittings near the electroweak scale in three-lepton final states in $\sqrt{s}=$ $13 \mathrm{TeV} p p$ collisions with the ATLAS detector," 1912.08479.

[17] ATLAS Collaboration, G. Aad et al., "Search for bottom-squark pair production with the ATLAS detector in final states containing Higgs bosons, $b$-jets and missing transverse momentum," JHEP 12 (2019) 060, 1908.03122.

[18] ATLAS Collaboration, G. Aad et al., "Search for electroweak production of charginos and sleptons decaying into final states with two leptons and missing transverse momentum in $\sqrt{s}=13 \mathrm{TeV} p p$ collisions using the ATLAS detector," Eur. Phys. J. C80 (2020), no. 2, $123,1908.08215$.

[19] ATLAS Collaboration, G. Aad et al., "Search for direct production of electroweakinos in final states with one lepton, missing transverse momentum and a Higgs boson decaying into two $b$-jets in (pp) collisions at $\sqrt{s}=13 \mathrm{TeV}$ with the ATLAS detector," 1909.09226 . 
[20] CMS Collaboration, V. Khachatryan et al., "Search for supersymmetry in the all-hadronic final state using top quark tagging in pp collisions at $\sqrt{s}=13 \mathrm{TeV}$," Phys. Rev. D96 (2017), no. 1, 012004, 1701.01954.

[21] CMS Collaboration, A. M. Sirunyan et al., "Search for supersymmetry in events with a $\tau$ lepton pair and missing transverse momentum in proton-proton collisions at $\sqrt{s}=13 \mathrm{TeV}$," JHEP 11 (2018) 151, 1807.02048.

[22] CMS Collaboration, A. M. Sirunyan et al., "Combined search for electroweak production of charginos and neutralinos in proton-proton collisions at $\sqrt{s}=13 \mathrm{TeV}$," JHEP 03 (2018) 160, 1801. 03957.

[23] CMS Collaboration, A. M. Sirunyan et al., "Search for top squarks decaying via four-body or chargino-mediated modes in single-lepton final states in proton-proton collisions at $\sqrt{s}=13 \mathrm{TeV}$," JHEP 09 (2018) 065, 1805.05784.

[24] CMS Collaboration, A. M. Sirunyan et al., "Search for Physics Beyond the Standard Model in Events with High-Momentum Higgs Bosons and Missing Transverse Momentum in Proton-Proton Collisions at $13 \mathrm{TeV}$," Phys. Rev. Lett. 120 (2018), no. 24, 241801, 1712.08501.

[25] CMS Collaboration, A. M. Sirunyan et al., "Search for supersymmetric partners of electrons and muons in proton-proton collisions at $\sqrt{s}=13 \mathrm{TeV}$," Phys. Lett. B790 (2019) 140-166, 1806.05264.

[26] CMS Collaboration, A. M. Sirunyan et al., "Searches for pair production of charginos and top squarks in final states with two oppositely charged leptons in proton-proton collisions at $\sqrt{s}=13 \mathrm{TeV}$," JHEP 11 (2018) 079, 1807.07799.

[27] CMS Collaboration, A. M. Sirunyan et al., "Search for supersymmetry in events with a photon, jets, $b$-jets, and missing transverse momentum in proton-proton collisions at $13 \mathrm{TeV}$," Eur. Phys. J. C79 (2019), no. 5, 444, 1901.06726.

[28] CMS Collaboration, A. M. Sirunyan et al., "Search for supersymmetry in proton-proton collisions at $13 \mathrm{TeV}$ in final states with jets and missing transverse momentum," JHEP 10 (2019) 244, 1908. 04722.

[29] CMS Collaboration, A. M. Sirunyan et al., "Search for supersymmetry in multijet events with missing transverse momentum in proton-proton collisions at 13 TeV," Phys. Rev. D96 (2017), no. 3, 032003, 1704.07781.

[30] W. Abdallah et al., "Reinterpretation of LHC Results for New Physics: Status and Recommendations after Run 2," 2003.07868.

[31] CMS Collaboration, S. Chatrchyan et al., "Interpretation of Searches for Supersymmetry with Simplified Models," Phys. Rev. D 88 (2013), no. 5, 052017, 1301. 2175.

[32] G. Chalons and H. Reyes-Gonzalez, "MadAnalysis 5 implementation of ATLAS-SUSY-16-07 (arXiv:1712.02332),".

[33] F. Ambrogi and J. Sonneveld, "MadAnalysis5 recast of CMS-SUS-16-033,".

[34] ATLAS Collaboration, "Reproducing searches for new physics with the ATLAS experiment through publication of full statistical likelihoods," Tech. Rep.

ATL-PHYS-PUB-2019-029, CERN, Geneva, Aug, 2019. https://cds.cern.ch/record/2684863.
[35] L. Heinrich, M. Feickert, and G. Stark, "pyhf: v0.4.1." https://github.com/scikit-hep/pyhf, DOI: 10.5281 /zenodo.1169739.

[36] G. Brooijmans et al., "Les Houches 2019 Physics at TeV Colliders: New Physics Working Group Report," in 11th Les Houches Workshop on Physics at TeV Colliders: PhysTeV Les Houches (PhysTeV 2019) Les Houches, France, June 10-28, 2019. 2019. 2002.12220.

[37] F. Ambrogi, S. Kraml, S. Kulkarni, U. Laa, A. Lessa, and W. Waltenberger, "On the coverage of the pMSSM by simplified model results," Eur. Phys. J. C78 (2018), no. 3, 215, 1707.09036.

[38] G. Chalons, M. D. Goodsell, S. Kraml, H. Reyes-González, and S. L. Williamson, "LHC limits on gluinos and squarks in the minimal Dirac gaugino model," JHEP 04 (2019) 113, 1812.09293.

[39] E. Conte, B. Fuks, and G. Serret, "MadAnalysis 5, A User-Friendly Framework for Collider Phenomenology," Comput. Phys. Comm. 184 (2013) 222-256, 1206.1599.

[40] B. Dumont, B. Fuks, S. Kraml, S. Bein, G. Chalons, E. Conte, S. Kulkarni, D. Sengupta, and C. Wymant, "Toward a public analysis database for LHC new physics searches using MADANALYSIS 5," Eur. Phys. J. C75 (2015), no. 2, 56, 1407. 3278.

[41] E. Conte and B. Fuks, "Confronting new physics theories to LHC data with MADANALYSIS 5," Int. J. Mod. Phys. A33 (2018), no. 28, 1830027, 1808.00480.

[42] J. Alwall, R. Frederix, S. Frixione, V. Hirschi, F. Maltoni, O. Mattelaer, H. S. Shao, T. Stelzer, P. Torrielli, and M. Zaro, "The automated computation of tree-level and next-to-leading order differential cross sections, and their matching to parton shower simulations," JHEP 07 (2014) $079,1405.0301$.

[43] T. Sjöstrand, S. Ask, J. R. Christiansen, R. Corke, N. Desai, P. Ilten, S. Mrenna, S. Prestel, C. O. Rasmussen, and P. Z. Skands, "An Introduction to PYTHIA 8.2," Comput. Phys. Commun. 191 (2015) 159-177, 1410. 3012.

[44] J. de Favereau, C. Delaere, P. Demin, A. Giammanco, V. Lemaître, A. Mertens, and M. Selvaggi, "DELPHES 3, A modular framework for fast simulation of a generic collider experiment," JHEP 02 (2014) 057, 1307.6346.

[45] ATLAS Collaboration, G. Aad et al., "Summary of the ATLAS experiment's sensitivity to supersymmetry after LHC Run 1 interpreted in the phenomenological MSSM," JHEP 10 (2015) 134, 1508.06608.

[46] http://hepdata.cedar.ac.uk/view/ins1389857. 